\title{
COMPARATIVO DOS EFEITOS ENTRE EVENTOS ENOS CANÔNICO E MODOKI NO PERÍODO DE 1980 A 2016, NO CENTRO-SUL DA AMÉRICA DO SUL
}

\author{
Valdeir Welter $^{(a)}$, Leila Limberger ${ }^{(b)}$ \\ (a) PPGG - Unioeste, Campus Marechal Cândido Rondon, valdeirwelter@gmail.com \\ (b) CCHEL - Unioeste, Campus Marechal Cândido Rondon, leila.limberger@ gmail.com
}

Eixo: Climatologia em diferentes níveis escalares: mudanças e variabilidades

\begin{abstract}
Resumo
Eventos climáticos no Cetro-sul da América do Sul são comumente relacionados à ocorrência de Eventos El Niño - Oscilação Sul (ENOS). O ENOS canônico é caracterizado pelo enfraquecimento (fortalecimento) dos ventos alísios e o aumento (diminuição) da Temperatura da Superfície do Mar (TSM) no Oceano Pacífico Equatorial Leste, originando o fenômeno El Niño (La Niña) (OLIVEIRA, 2001). Ocasionalmente, a mudança de TSM pode ocorrer com outro padrão espacial, na região do Pacífico Central, chamado de ENOS Modoki (ASHOK et al., 2007), ou "imitação" em japonês. Esta pesquisa objetiva ampliar os estudos sobre os efeitos dos ENOS Modoki, propondo-se a identificá-los e compará-los as ENOS Canônico, observando as implicações sobre a precipitação no Centro-Sul da América do Sul. Espera-se contribuir para o entendimento da variabilidade climática ocasionada pelos diferentes tipos de eventos ENOS, ampliando a compreensão de que cada evento ENOS é único (AMBRIZZI, 2003) e que possuem efeitos variáveis espacialmente.
\end{abstract}

Palavras chave: ENOS, Canônico, Modoki, anomalias, Centro-sul da América do Sul.

\section{Introdução}

A atmosfera ainda pode ser considerada o domínio mais pulsante, dinâmico e imprevisível de todas as esferas naturais do planeta (SANT'ANNA NETO, 2001, p. 57), se configurando como uma das fronteiras para o pensamento geográfico.

Segundo Sant'anna Neto (1998, p.123)

Na concepção geográfica, o papel do clima na organização do espaço deve ser visto, fundamentalmente, como gerador de tipos de tempo cujas características são absolutamente dinâmicas, complexas e muito sensíveis a qualquer alteração imposta, influenciando cada parte do planeta, em função da interação entre as diferentes esferas do globo e da ação do homem.

Nesse sentido, devido as constantes variabilidades temporais decorrentes de eventos climáticos na região centro-sul da América do Sul, comumente relacionados à ocorrência de eventos Niños (ENOS) (GRIMM,

DOI - 10.20396/sbgfa.v1i2017.2436 - ISBN 978-85-85369-16-3 
FERRAZ \& GOMES, 1998; GRIMM, BARROS \& DOYLE, 2000; GRIMM, 2004; CATALDI, 2008; TEDESCHI, 2008; TEDESCHI \& CAVALCANTI, 2010; PBMC, 2012), se faz necessário um estudo aprofundado desta dinâmica, auxiliando em ações de projeção, prevenção e de respostas rápidas aos efeitos decorrentes desses episódios.

As condições que indicam a presença do fenômeno ENOS conhecido como Canônico ou Convencional são o enfraquecimento (intensificação) dos ventos alísios e o aumento (diminuição) da Temperatura da Superfície do Mar (TSM) no Oceano Pacífico Equatorial Leste, acarretando na diminuição (aumento) das águas mais frias que afloram próximo à costa oeste da América do Sul (OLIVEIRA, 2001).

Porém, além da existência de um El Niño Convencional (Canônico), onde o máximo de anomalia de TSM ocorre na região do Pacífico Leste Equatorial (PBMC, 2012), há a presença de uma outra estrutura espacial, intitulada El Niño Modoki (ASHOK et al., 2007) ou Central (McPHADEN, ZEBIAK \& GLANTZ, 2006), onde o máximo das anomalias de TSM ocorre no Pacífico Central.

Observando esses conjuntos de elementos, é possível perceber alguns eventos registrados pelo mundo como consequência de El Niño canônico, como secas na região nordeste do Brasil e cheias provocadas por grandes volumes de precipitação no cone sul da América do Sul (OLIVEIRA, 2001, CATALDI, 2008, TEDESCHI \& CAVALCANTI, 2010, RODRIGUES et al. 2012), mas pesquisas acerca da influência do El Niño Modoki sobre a América do Sul ainda são escassas, possivelmente porque esses impactos devem ser mais fracos (PBMC, 2012).

A presente pesquisa tem por objetivo ampliar os estudos sobre os efeitos dos ENOS Modoki, propondo-se a identificar e comparar os efeitos de ENOS Canônico e Modoki sobre a precipitação no Centro-Sul da América do Sul. Espera-se, com isso, contribuir para o entendimento da variabilidade climática ocasionada pelos diferentes tipos de eventos ENOS, ampliando a compreensão de que cada evento ENOS é único (AMBRIZZI, 2003) e que possuem efeitos variáveis espacialmente, estando em estágio inicial de pesquisa.

\section{Referencial Teórico}

Um dos fenômenos climáticos amplamente estudados pela comunidade acadêmica sem dúvida é o El Niño e suas variabilidades.

Segundo Oliveira (2001),

DOI - 10.20396/sbgfa.v1i2017.2436 - ISBN 978-85-85369-16-3 


\section{OS DESAFIOS DA GEOGRAFIA FÍSICA NA FRONTEIRA DO CONHECIMENTO \\ Instituto de Geociências - Unicamp \\ Campinas - SP \\ 28 de Junho à 02 de Julho de 2017}

El Niño representa o aquecimento anormal das águas superficiais e sub-superficiais do

Oceano Pacífico Equatorial [...]. Na atualidade, as anomalias do sistema climático que são mundialmente conhecidas como El Niño e La Niña representam uma alteração do sistema oceano-atmosfera no Oceano Pacífico tropical, e que tem consequências no tempo e no clima em todo o planeta. Nesta definição, considera-se não somente a presença das águas quentes da Corriente El Niño mas também as mudanças na atmosfera próxima à superfície do oceano, com o enfraquecimento dos ventos alísios (que sopram de leste para oeste) na região equatorial. Com esse aquecimento do oceano e com o enfraquecimento dos ventos, começam a ser observadas mudanças da circulação da atmosfera nos níveis baixos e altos, determinando mudanças nos padrões de transporte de umidade e, portanto, variações na distribuição das chuvas em regiões tropicais e de latitudes médias e altas. Em algumas regiões do globo também são observados aumento ou queda de temperatura.

Este fenômeno de alcance global também pode ser definido como,

[...] um fenômeno no Oceano Pacífico equatorial, caracterizado por um desvio de temperatura da superfície do mar positivo além do normal (para o período base de 19712000) na região Niño 3.4 [...] maior ou igual em magnitude a $0,5^{\circ} \mathrm{C}$, em média, por mais de três meses consecutivos. (ASHOK et al, 2007, tradução livre)

O oceano Pacífico, na altura da Linha do Equador, é dividido em regiões (figura 1) que possibilitam a identificação correta do fenômeno, como citado acima, onde variações da TSM são constatadas e registradas.

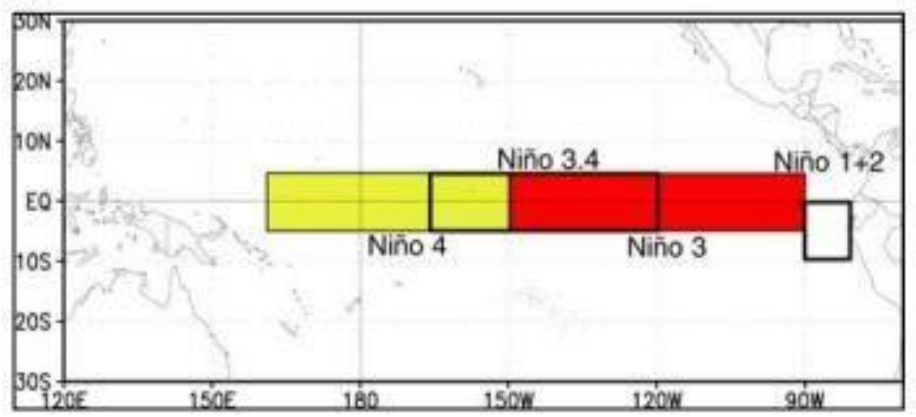

Figura 1 - Áreas de atuação do El Niño sobre o pacífico tropical. (Adaptado de Oliveira, 2001).

Partindo desse princípio, o Índice Oceânico do Niño (ION) foi desenvolvido, tornando-se o padrão que o NOAA (National Oceanic and Atmospheric Administration, E.U.A.) usa para identificar El Niño (aquecimento) e eventos de La Niña (resfriamento) no Pacífico tropical. Ele significa a ocorrência de anomalia de TSM por três meses consecutivos para a região Niño 3.4 (vide figura 1). Os eventos são então

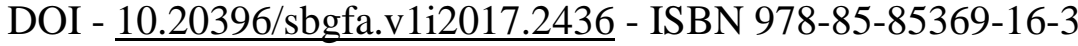




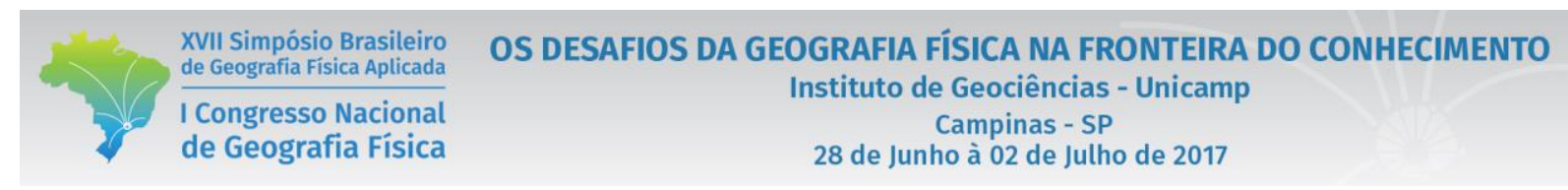

definidos por meio de cinco consecutivas sobreposições de períodos de três meses com anomalia superior a $0,5^{\circ}$ mais quente (El Niño) ou abaixo de $0,5^{\circ}$ mais frio (La Niña) do que a média normal da TSM (GGWS, 2016).

Normalmente, os eventos são classificados de acordo com sua intensidade, onde a variação do ION é levada em consideração, como mostra a tabela (I) abaixo.

Tabela I - Critérios para classificar a intensidade dos fenômenos El Niño e La Niña (GGWS, 2016).

\begin{tabular}{|l|l|l|}
\hline Evento & Valor do ION & Intensidade \\
\hline \multirow{4}{*}{ EI Niño } & 0,5 a 0,9 & Fraca \\
& 1,0 a 1,4 & Moderada \\
& 1,5 a 1,9 & Forte \\
& $\geq 2,0$ & Muito forte \\
\hline \multirow{4}{*}{ La Niña } & $-0,5$ a $-0,9$ & Fraca \\
& $-1,0$ a $-1,4$ & Moderada \\
& $-1,5$ a $-1,9$ & Forte \\
& $\leq-2,0$ & Muito forte \\
\hline
\end{tabular}

Porém, foram identificadas variações na própria anomalia, pois conforme a predominância de temperatura mais alta na região Niño 3.4 em detrimento das outras, um fenômeno diferente ocorre. O El Niño Canônico já não explica mais as consequências desencadeadas dessa variação, surgindo então a expressão El Niño Modoki.

Ashok et. al. (2007) definem essa subdivisão, onde eventos Canônicos e Modoki se diferem principalmente no inverno austral, notadamente com a falta do precursor aquecimento (resfriamento) da costa do Peru no caso do "El Niño (La Niña) Modoki", palavra que possui vários significados como "falso, simulado ou imitação" em japonês.

A condição normal de TSM na região central do oceano Pacífico é a concentração de águas quentes na parte oeste e de águas frias na região leste. A essa condição normal das águas do oceano Pacífico denomina-se de ano Neutro (GRIMM, FERRAZ \& GOMES, 1998). O evento Modoki é caracterizado então por anomalias positivas de temperatura da superfície do mar (TSM) no Pacífico Central acompanhado por anomalias negativas de TSM no Pacífico Leste e Oeste (TEDESCHI \& CAVALCANTI, 2010), enquanto no evento canônico o máximo de anomalia de TSM ocorre na região do Pacífico Leste Equatorial (PBMC, 2012).

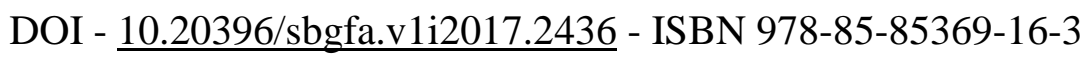


Como ocorrem em locais diferentes do Pacífico, os dois fenômenos têm efeitos também diferentes ao redor do globo. O ENOS canônico acarreta algumas anomalias meteorológicas no Brasil, como secas na região nordeste e cheias provocadas por grandes volumes de precipitação no cone sul da América do Sul (OLIVEIRA, 2001; CATALDI, 2008; TEDESCHI \& CAVALCANTI, 2010; RODRIGUES et al. 2012). Durante eventos ENOS Modoki os efeitos sobre a América do Sul parecem acarretar La Niñas mais fortes e El Niños deslocados, segundo Kao \& Yu (2009).

As anomalias são percebidas por meio de coleta de dados meteorológicos, de precipitação, direção dos ventos, umidade, TSM, dentre outros, que hoje, com a democratização de dados meteorológicos por meio de processos simples de download a partir de grandes centros de pesquisa mundiais (LIMBERGER, 2015), vem contribuindo para a multiplicação das correlações entre essas informações.

Dados de Reanálise são hoje fontes importantes para a observação das anomalias. Esses conjuntos de elementos são utilizados para estudos que analisam a variabilidade temporal ou espacial de variados fenômenos atmosféricos, tendências, médias, etc., cujos projetos principais são o NCEP/NCAR I e NCEP/DOE, o Global Precipitation Climatology Centre (GPCC) e também o Climate Research Unit (CRU) (LIMBERGER, 2015).

Por meio dessas coletâneas de informações, é possível estabelecer teleconexões entre os fenômenos ENOS e seus resultados no globo, em especial no centro-sul da América do Sul, que sofre consequências bem marcadas quando da ocorrência de ENOS. Teleconexão, por sua vez, pode ser definida como a relação de causa e efeito entre processos ambientais que guardam distância entre si, onde forçantes locais agem para influenciar regiões remotas e vice-versa (AMBRIZZI 2003; McPHADEN, ZEBIAK \& GLANTZ, 2006; CATALDI, 2008).

Quanto à precipitação, suas quantidades médias mensais ou sazonais tendem a aumentar durante as fases do El Niño na região sul do Brasil e da América do Sul, assim, espera-se que a frequência de eventos extremos de chuva seja impactado pelo El Niño em alguns locais. Entretanto, isso não significa que regiões que sofrem a influência do mesmo sobre a precipitação mensal e sazonal sofrerão necessariamente influência sobre a frequência e intensidade dos eventos extremos (TEDESCHI, 2008).

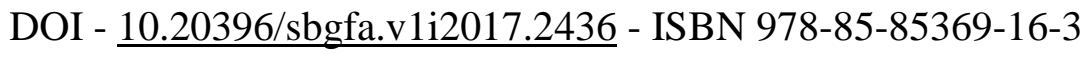




\section{Materiais e Métodos}

A fim de buscar uma ligação entre anomalias de precipitação no Centro-sul da América do Sul e o fenômeno ENOS, o método da teleconexão atmosférica foi utilizado, já amplamente difundido na Meteorologia.

Uma das principais fontes para a obtenção de dados climáticos foram os resultados de Reanálise ou reconstrução de dados, resultado do agrupamento de várias fontes primárias, tais como satélites meteorológicos, estações de superfície, plataformas de coletas de dados, etc. (LIMBERGER, 2015).

Os dados de TSM utilizados são os da Reanálise NCEP/NCAR (KALNAY et al., 1996) e os dados de precipitação serão do Global Precipitation Climatology Centre (GPCC) e do Climate Reseach Unit (CRU), sendo estes conjuntos de dados organizados por órgão e universidades ao redor do globo como NOAA (National Oceanic and Atmospheric Administration, E.U.A.) e o ECMWF (European Centre for MediumRange Weather Forecasts).

Dados de precipitação e TSM de anos com eventos ENOS foram especializados com o auxílio do site esrl.noaa.gov.br e a ferramenta "Interact Plotting", o que possibilita conhecer os dados e identificar em quais anos houve ocorrência de eventos Canônico e Modoki e como se configurou a distribuição espacial da precipitação para a América do Sul.

\section{Resultados e Discussão}

A interpolação dos dados de reanálise tem permitido destacar ENOS marcantes que ocorreram recentemente, tanto das variantes canônica quanto Modoki.

A tabela (II) abaixo permite posicioná-los de forma a perceber que as anomalias de precipitação positivas (negativas) são marcadas no centro-sul da América do Sul em ocorrências de ENOS canônicos, enquanto em decorrência de aquecimento (resfriamento) marcado no Pacífico central, sem os episódios de ressurgência característica na costa oeste da região equatorial da América, há um deslocamento ou inversão das anomalias de precipitação. 


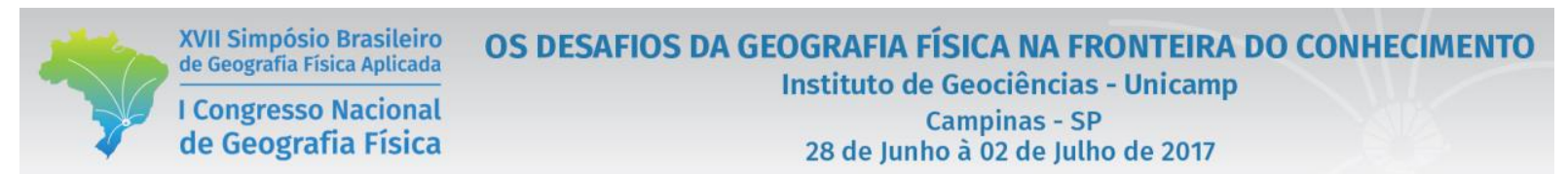

Tabela II - Eventos ENOS: anomalias de precipitação correspondente para a América do Sul: (a) anomalias de TSM

$\left({ }^{\circ} \mathrm{K}\right)$ global para o período de dezembro de 1997 a fevereiro de 1998; (b) anomalia de precipitação (mm/dia) para o período de dezembro de 1997 a fevereiro de 1998 na América do Sul; (c) anomalias de TSM $\left({ }^{\circ} \mathrm{K}\right)$ global para o período de dezembro de 1991 a fevereiro de 1992; (d) anomalia de precipitação (mm/dia) para o período de dezembro de 1991 a fevereiro de 1992 na América do Sul; (e) anomalias de TSM $\left({ }^{\circ} \mathrm{K}\right)$ global para o período de junho a agosto de 1988; (f) anomalia de precipitação (mm/dia) para o período de junho a agosto de 1988 na América do

Sul; (g) anomalias de TSM ( $\left.{ }^{\circ} \mathrm{K}\right)$ global para o período de junho a agosto de 1999; (h) anomalia de precipitação (mm/dia) para o período de junho a agosto de 1999 na América do Sul.

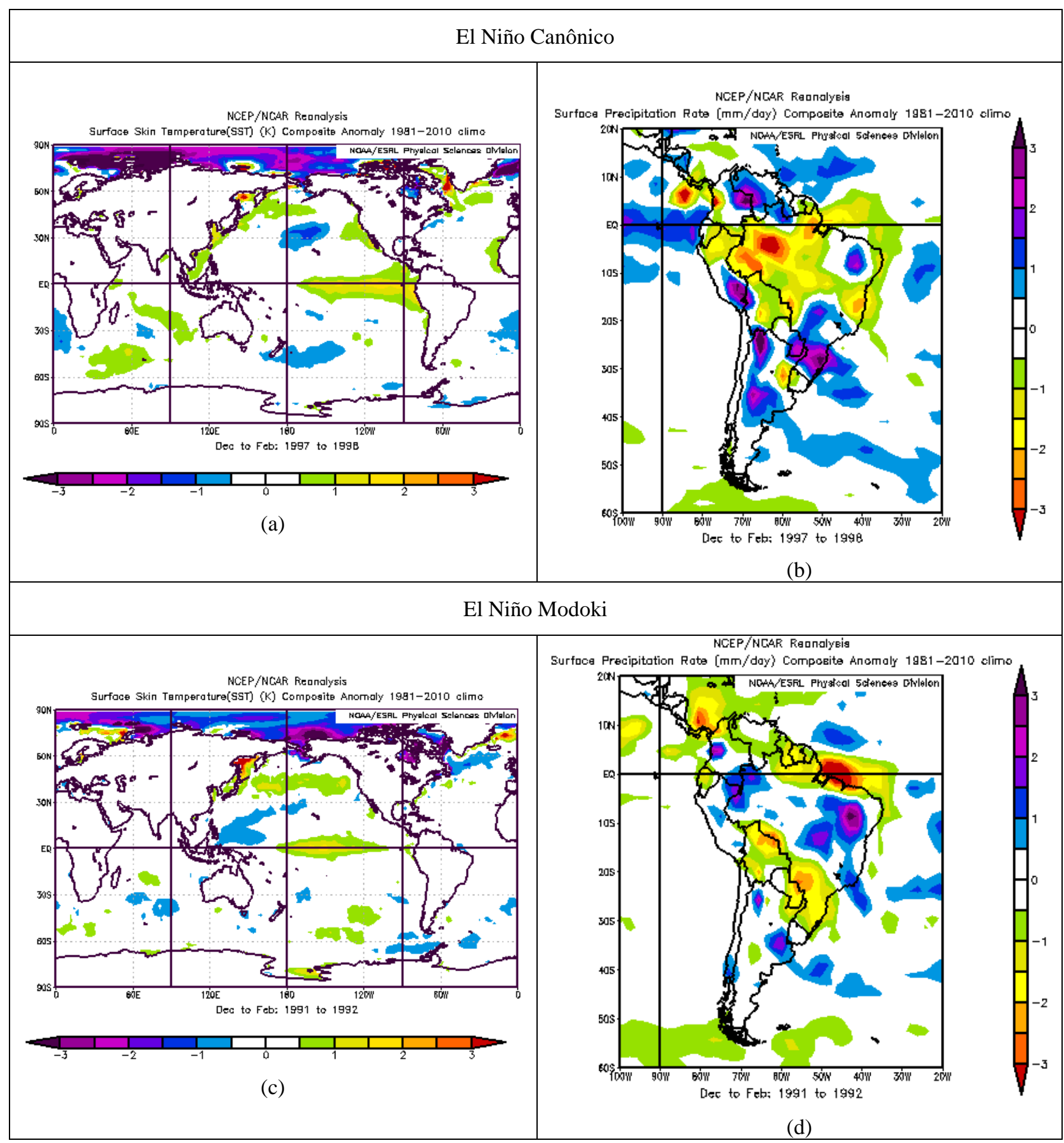




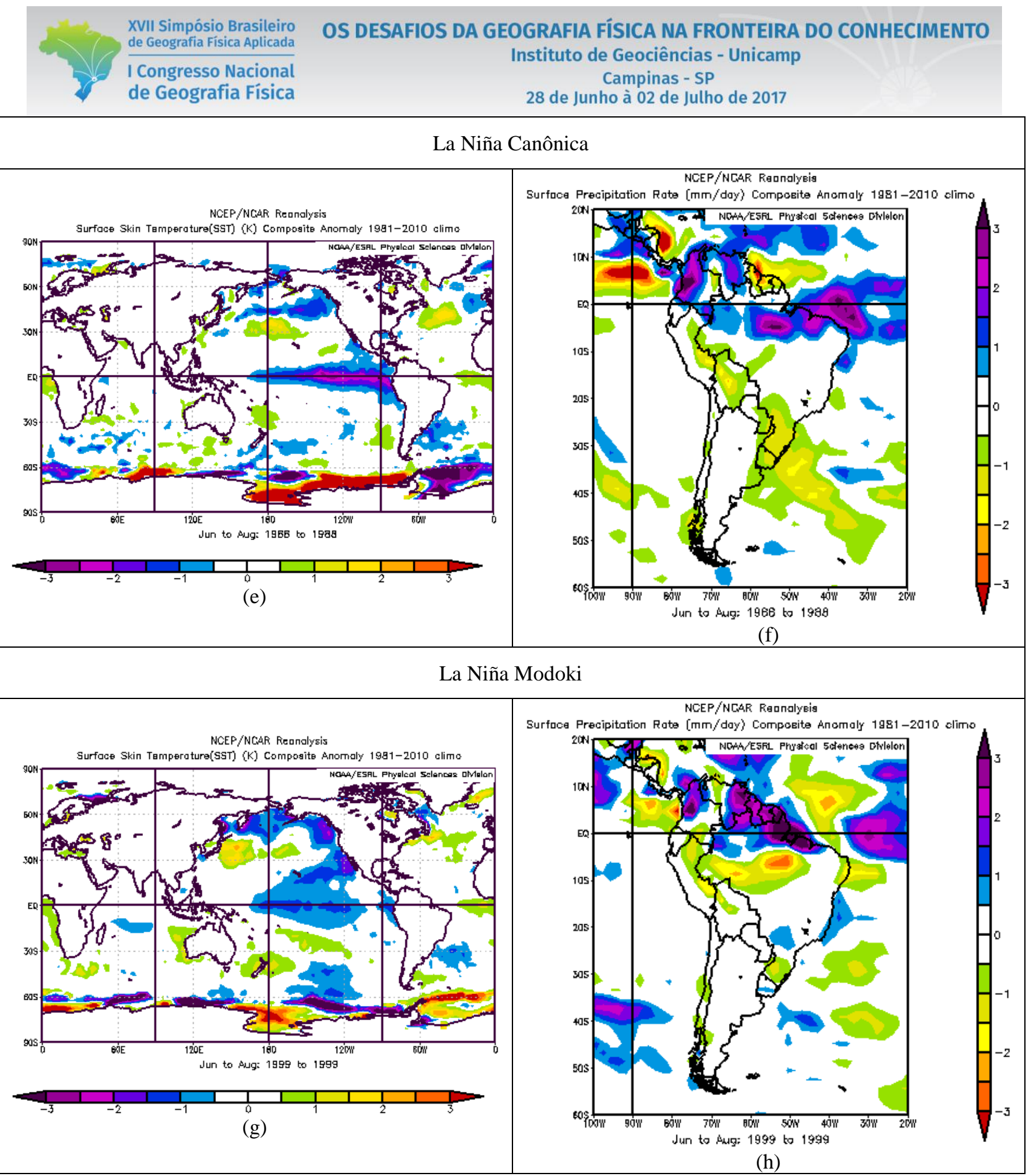

Na região centro-sul da América do Sul é aparente a inversão do sinal de anomalia de precipitação em (d), uma vez que com o aquecimento do pacífico o esperado seria um incremento no volume de chuvas, enquanto em (h), observa-se além da inversão também um deslocamento do sinal de seca para o Atlântico.

Comparando outros episódios ENOS, observa-se em 2015/16 a ocorrência de um El Niño forte, superando o evento de 1982/83 segundo o ION, sendo esse o índice que identifica as anomalias da superfície do 
Oceano Pacífico através de uma média móvel de três meses, e que desde abril de 2014 vem o caracterizando com força muito semelhantes ao El Niño canônico de 1997, período entremeado por vários El Niños Modoki (PBMC, 2012), oportunizando um estudo aprofundado de comparação de suas consequências sobre o sul do Brasil, norte da Argentina, Uruguai e Paraguai (GRIMM, BARROS \& DOYLE, 2000, GRIMM et al, 1998, GRIMM, 2003, 2004), que acarretam grandes desastres como cheias, deslizamentos de terra e prejuízos nas safras.

\section{Considerações finais}

Para aprofundar a presente pesquisa, mapas de correlação linear entre TSM e precipitação no Centro-Sul da América do Sul serão confeccionados por meio do software $\mathrm{NCL}^{\oplus}$. Com esses mapas de correlação será possível detectar quais áreas oceânicas mais influenciam na variabilidade da chuva na área de estudo. Para verificar os efeitos dos dois tipos de eventos ENOS, serão levantados na bibliografia e por meio de análise de série temporal mensal a TSM do Pacífico no período de 1980 a 2016, identificando quais os anos em que se configuraram eventos ENOS Canônicos ou Modoki.

Além dos dados de precipitação, também serão confeccionados, para o mesmo período e escala, mapas das principais variáveis atmosféricas, tais como vetor do vento, convergência de umidade e velocidade vertical do vento. Com isso será possível explicar os motivos das anomalias positivas ou negativas de precipitação.

\section{Bibliografia}

The El Niño Impact on Summer Monsoon in Brazil: Regional Processes versus Remote Influences. Journal of Climate, v. 16, p. 263-280, 2003.

The NCAR Command Language (Version 6.3.0) [Software]. (2015). Boulder, Colorado: UCAR/NCAR/CISL/TDD.<http://dx.doi.org/10.5065/D6WD3XH5>

BARROS, V. R. e DOYLE, M. E. Climate variability in Southern South America associated with El Niño and La Niña events. Journal of Climate, v. 13, p. 35-58, 2000. 138, 2004.

How do La Niña events disturb the summer monsoon system in Brazil? Climate Dynamics, 22, n.2-3, 123-

Por uma geografia do clima: antecedentes históricos, paradigmas contemporâneos e uma nova razão para um novo conhecimento. Terra Livre. São Paulo, n. 17, $2^{\circ}$ semestre/2001. p. 49-62.

AMBRIZZI, T. El Niño Oscilação Sul e Teleconexões atmosféricas no hemisfério austral. São Paulo: USP/IAG, 2003. (Tese de Livre-Docência).

ASHOK, K., BEHERA, S. K., RAO, S. A., WENG, H. \& YAMAGATA, T. (2007). El Niño Modoki and its possible teleconnection. J. Geophys. Res., 112,. C11007, doi:10.1029/2006JC003798.

CATALDI, M. Estudo numérico da influência das anomalias da TSM do Atlântico Sul extratropical e do Pacífico Equatorial no regime hidrometeorológico das regiões Sul e Sudeste do Brasil. (2008). 203 f. Tese

DOI - 10.20396/sbgfa.v1i2017.2436 - ISBN 978-85-85369-16-3 


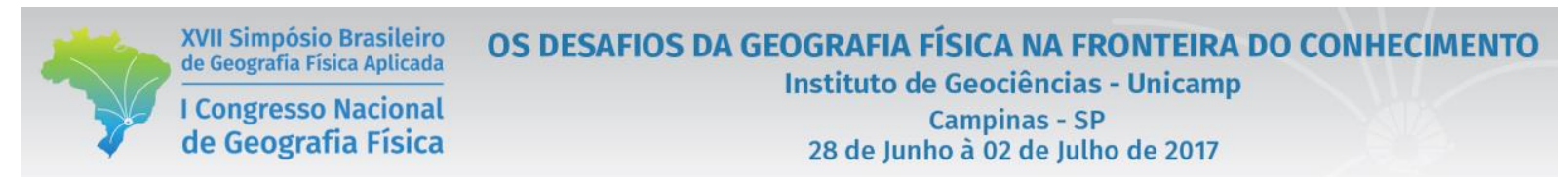

(Doutorado) - Curso de Engenharia, Coppe/ufrj, D.sc., Engenharia Civil, Universidade Federal do Rio de Janeiro, Rio de Janeiro, $2008 . \quad$ Disponível em: <http://numa.lamce.coppe.ufrj.br/DATA/formacao/CATALDI_2008_DSc.pdf>. Acesso em: 10 jan. 2016.

GRIMM, A, FERRAZ, S. E. T. \& GOMES, J. Precipitation anomalies in Southern Brazil associated with El Niño and La Niña events. J. Climate, v. 11, p. 2863-2880. 1998.

KALNAY et al., The NCEP/NCAR 40-year reanalysis project, Bull. Amer. Meteor. Soc., 77, 437-470, 1996.

KAO, H. -Y.; YU, J. -Y. Contrasting Eastern-Pacific and Central Pacific types of ENSO. Journal of Climate. Vol. 22, p. 615-632, fev. 2009.

LIMBERGER, L. Correlação linear entre conjuntos de dados de chuva para a Amazônia brasileira. In: IX Expedição Geográfica da Unioeste: Geografia Política e Ambiente, 2015, Marechal Cândido Rondon. v. 1. p. 1-4.

MCPHADEN, M. J., ZEBIAK S. E., GLANTZ, M. H. ENSO as an integrating concept in Earth Science. Science, 314, 1740-1745, 2006. doi:10.1126/science.1132588.

OLIVEIRA, G. S. de. O El Niño e Você - o fenômeno climático. Editora Transtec - São José dos Campos (SP), março de 2001 .

PBMC. Sumário Executivo do Volume 1 - Base Científica das Mudanças Climáticas. Contribuição do Grupo de Trabalho 1 para o 1 o Relatório de Avaliação Nacional do Painel Brasileiro de Mudanças Climáticas. Volume Especial para a Rio+20. PBMC, Rio de Janeiro, Brasil, 34 p. 2012.

RODRIGUES, R. R., HAARSMA R.J., CAMPOS E.J.D., AMBRIZZI T. The impacts of interEl Niño Variability on the tropical Atlantic and Northeast Brazil climate. Journal of Climate, 24: 3402-3422. 2012. doi: http://dx.doi.org/10.1175/2011JCLI3983.1.

SANT'ANNA NETO, J. L. Clima e organização do espaço. Boletim de Geografia, n. 16: 119-131, Maringá, 1998. TEDESCHI, R. G. Impacto de episódios el niño e la niña sobre a frequência de eventos extremos de precipitação e vazão na América do Sul. 159 f. Dissertação (Mestrado) - Curso de Pós-graduação em Engenharia de Recursos Hídricos, Setor de Tecnologia, Universidade Federal do Paraná, Curitiba, 2008.

TEDESCHI, R. G.; CAVALCANTI, I. F. A. Influência dos ENOS Canônico e Modoki na precipitação da América do Sul. In: CONGRESSO BRASILEIRO DE METEOROLOGIA, 16. 2010. Belém do Pará - PA. Anais ... Belém do Pará-PA, 2010.

DOI - 10.20396/sbgfa.v1i2017.2436 - ISBN 978-85-85369-16-3 